\title{
UN CONCEPTO RARÁMURI DE CUERPO. EXPERIENCIA PRÓXIMA E INTERPRETACIÓN DISTANTE
}

The Rarámuri bODY IN THE Upper Río Conchos. NEAR EXPERIENCE AND DISTANT INTERPRETATION

ABEL RODRÍGUEZ LÓPEZ

\section{RESUMEN}

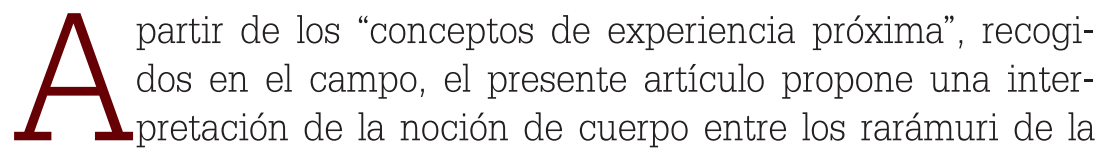
región conocida como Alto Río Conchos y, posteriormente, una interpretación de "experiencia distante". Lo que intento probar es que el cuerpo rarámuri no solo se concibe como origen de representaciones del cosmos ni tampoco únicamente como un producto de representaciones sociales e históricas, sino que es considerado en ambos sentidos. Asimismo, sugiero que el cuerpo rarámuri puede también ser interpretado siguiendo la idea del eje “arriba” y "abajo".

Palabras clave: cuerpo, rarámuri, sierra Tarahumara, interpretación.

1 Docente investigador en la Universidad Autónoma de San Luis Potosí, México. Correo electrónico: ta_abeli@hotmail.com 


\section{ABSTRACT}

From the "experience-near concepts", collected in field, this paper proposes an interpretation of body concept in the rarámuri thinking from the region known as the Upper Río Conchos; then it proposes an interpretation of "experience-distant". What the author tries to prove is that rarámuri conception not only conceived the body as a source of representations of the cosmos nor only as a consequence of social and historical representations, but it is considered in both directions. Besides suggests that body rarámuri can be interpreted under the axis "up" and "down".

Keywords: body, rarámuri, sierra Tarahumara, interpretation.

La observación etnográfica de la concepción del tiempo y el espacio de los rarámuri del Alto Río Conchos, México (en adelante ARC), junto con la consideración de la terminología referida al espacio y el tiempo en relación con otros aspectos de la vida cotidiana (trabajo, ritualidad, mitos), y la reiterativa orientación del eje "hacia arriba"-"hacia abajo", me remitieron una y otra vez a las nociones de cuerpo, persona y sociedad (Rodríguez, 2015). Por otra parte, en una de mis estancias de trabajo de campo al preguntar a algunos rarámuri sobre las partes del cuerpo en su lengua, llamó mi atención que al hueso saliente del tobillo, así como al de la muñeca los llamaran bachakó. Mi interés aumentó cuando caí en la cuenta de que a los nudos de las cañas y de los árboles, se les llama del mismo modo: bachakó. Este hecho me motivó a buscar otras coincidencias del mismo tipo, lo que suscitó la pregunta por el concepto de cuerpo entre los rarámuri. Más adelante descubrí que el término wichí, que los rarámuri emplean para referirse a la "piel" humana y animal, también es empleado para referirse al "suelo" y, en general, a la cubierta de cualquier objeto. Posteriormente, algunos topónimos como Banarachi (lugar de caras), Sekachiki (lugar de manos) o Bisawiríachi (lugar de los penes en pie) y otros, me llevaron a la pregunta sobre si el cuerpo humano es un referente para entender el entorno. Con base en lo anterior, me pregunto ahora: ¿cuál es la noción de cuerpo entre los rarámuri del 
Un CONCEPTO RARÁMURI DE CUERPO. EXPERIENCIA PRÓXIMA E INTERPRETACIÓN...

ARC?, noción en tanto código de entendimiento en las relaciones sociales de una parte determinada del grupo.

Los estudios sobre las nociones de cuerpo y persona entre los rarámuri de la sierra Tarahumara son escasos. Existen algunas tesis como las de Guillén y Martínez (2004) y Martínez (2008) que han desarrollado el concepto de "cuerpo" en relación con ideas como el "caminar bien" y el "caminar del sol" en tanto ideas propias de la ética rarámuri. Por su parte, Acuña (2007; 2009), desde la perspectiva del simbolismo corporal, abordó la construcción cultural del cuerpo y presenta un modelo que resalta, de modo central, la singularidad del comportamiento motriz en materia de las danzas y la carrera pedestre de resistencia, así como de la vida cotidiana y algunos momentos rituales. Al estudiar el cuerpo en movimiento, Acuña (2007) destaca algunos rasgos distintivos de la corporeidad rarámuri como la complementariedad y el sentido práctico vividos entre hombres y mujeres, dejando su análisis en el umbral de los estudios de género. El autor concluye que "en el cuerpo se perciben las huellas del proceso de cambio cultural, las consecuencias del choque o contactos con otras culturas" (p. 442), sugiriendo así que el cuerpo, si bien es parte de un hecho biológico, debe ser pensado, también, como el resultado de los procesos históricos y sociales.

Siguiendo esta última idea, mi objetivo es mostrar que, efectivamente, la concepción del cuerpo rarámuri no es estática y que más bien se trata de una visión en la que el cuerpo se concibe como producto de las relaciones sociales e históricas, pero también como el origen de representaciones del cosmos. Además, sugiero que el cuerpo puede ser interpretado desde la idea reiterativa del eje "arriba"-abajo".

Siguiendo a Le Bretón (2002) no examinaré el cuerpo rarámuri “como una realidad en sí misma” (p. 13), porque creo que realmente el cuerpo como tal no tiene existencia y, como lo sugieren mis datos, tampoco es una construcción consciente y determinada. Por el contrario, abordaré el cuerpo, por una parte, como producto de representaciones e imposiciones sociales y culturales (Foucault, 2014 [1966]; 1976); por otra parte, la observación me remitió 
también a la consideración que hace ya tiempo Douglas (1973) hiciera, al señalar que el cuerpo es una fuente de representaciones del mundo, algo similar a lo que mucho antes Durkheim y Mauss (1971 [1903]) habían señalado acerca de que las clasificaciones humanas preceden a las de la naturaleza.

Una forma que encuentro pertinente para llevar a cabo el abordaje de esta noción es mediante el análisis de algunos conceptos de experiencia próxima, que puedan luego situarnos en posición de hablar de un modelo de experiencia distante o teórico. De modo general diré aquí que los conceptos de experiencia próxima son aquellos que se emplean de manera coloquial y sin mayor esfuerzo para definir "lo que un sujeto cualquiera ve, siente, piensa, imagina, etc., y que podría comprender con rapidez en el caso de que fuese aplicado de forma similar por otras personas" (Geertz, 2004, p. 75). Por otro lado, los conceptos de experiencia distante serían aquellos que un científico emplea para impulsar sus propósitos, partiendo, claro está, de los primeros. De este modo, me propongo mostrar aquí los significados de las cosas que vienen del uso que se les da, porque "sólo analizando esos usos [...] seremos capaces de averiguar algo sobre ellos” (ibidem, pp. 144-145). ¿A qué usos me refiero?, a los que llevan a cabo en la cotidianidad de sus vidas las personas que se relacionan entre sí, con su entorno e, incluso, con su mundo no perceptible.

En este trabajo, además, me inspiro en una de las principales ideas de Sapir (1956), quien consideró el lenguaje como el resultado de un proceso cultural y social, y planteó que para obtener una mejor comprensión de un grupo humano hay que aproximarse a los puntos de intersección entre el pensamiento, la lengua y las acciones sociales. Concuerdo con Sapir (1956) en que el lenguaje no determina, pero sí es condicionante de algunas prácticas en las relaciones sociales. Mi aproximación en el campo, pues, fue hecha observando el sistema lengua-cultura y desde el presupuesto bourdieano de que "el lenguaje común y ciertos usos especializados de las palabras comunes constituyen el principal vehículo de las representaciones comunes de la sociedad” (Bourdieu, 2008, p. 32). 
Un CONCEPTO RARÁMURI DE CUERPO. EXPERIENCIA PRÓXIMA E INTERPRETACIÓN...

Los datos presentados en este artículo han sido recabados mediante la observación participante y la convivencia con hombres jóvenes y adultos, especialmente, debido a la dificultad que representa para una persona no indígena y externa dialogar con las mujeres de la comunidad y viceversa. ${ }^{2}$ Los ámbitos de observación han sido los de la vida cotidiana en actividades colectivas, visitas en las viviendas de algunas familias y algunos espacios rituales. Asimismo, realicé entrevistas abiertas con algunas autoridades de las comunidades rarámuri y tres owirúame (lit. "los que dicen curan"), especialistas rituales practicantes de ceremonias curativas, ante todo. Al principal de ellos lo llamo aquí José María.

Antes de hablar de las relaciones entre el cuerpo y el cosmos, la sociedad y la historia, y una serie de otros aspectos culturales que me ayudarán a llevar a cabo mi ejercicio interpretativo, diré algo sobre quiénes son los rarámuri (endoetnónimo) o tarahumaras (castellanismo) de México.

\section{LOS TARAHUMARAS O RARÁMURI}

Según cifras del Inegi (2015), actualmente viven unos 73856 hablantes de la lengua rarámuri. Los rarámuri, o tarahumaras, del ARC son agricultores, pastores de ganado caprino y vacuno en baja escala, artesanos y jornaleros. Sus principales cultivos y alimentos son calabaza, frijol y maíz, pero la dieta se complementa con la caza, recolección y pesca (en algunos sitios donde pasan afluentes del río Conchos) en un hábitat propio del bosque de coníferas. Con el maíz preparan el tesgüino, cerveza local empleada en toda reunión comunitaria de trabajo colectivo y las prácticas religiosas. Algunos hombres producen instrumentos musicales, herramientas de trabajo y otros utensilios de madera, y algunas mujeres fabrican cestos, ollas, ropa, cobijas, etcétera, a base de palmilla, barro e hilos o lana. En épocas previas a la siembra y la cosecha, hay quienes se emplean en la recolección de manzana o tomate, en

2 La etnografía aquí presentada es el resultado de distintas estancias en el ARC, especialmente en los ejidos rarámuri de Tewerichi, Narárachi, Chinéachi, Bakiachi (entre 2014 y 2016), y otras estancias de años atrás, apoyado por los misioneros redentoristas del municipio de Carichí, Chih. A ellos agradezco especialmente su hospitalidad.

Chimuahua Hoy, aÑo i6, Núm. I6 (ENERo-Diciembre, 2018) 
ranchos menonitas y mestizos, en la construcción de viviendas y hasta como mecánicos, traductores o empleadas domésticas en las cabeceras municipales más pobladas de la sierra o en las principales ciudades del estado de Chihuahua.

El primer registro que se tiene de ellos es del jesuita catalán Joan Font, quien en 1607 describió a los "taraumaros" como dóciles al cristianismo (cit. en González, 1982, p. 155; 1987, p. 147). A pesar de mantener fuerte su creencia en Onorúame ("el que dicen es Padre", asociado con el sol) y Eyerúame ("la que dicen es Madre”, asociada con la luna), adoptaron la Semana Santa y las celebraciones católicas del 12 de diciembre y el 6 de enero, ${ }^{3}$ en particular, durante las cuales ejecutan danzas que poco tienen que ver con la ortodoxia romana y donde (en Semana Santa) sobresale una "lucha" entre el bien y el mal. Los rarámuri son conocidos por su práctica de las carreras de bola, que llevan a cabo los hombres, y de aros las mujeres. Estas carreras, sobre todo la de hombres, pueden durar hasta un día completo. Un explorador noruego queriendo encontrar a los últimos cave dwellers describió a los rarámuri de finales del siglo XIX como los corredores de más resistencia en el mundo (Lumholtz, 1981 [1902], p. 297) y tanto en el siglo xx como más actualmente, los etnógrafos siguen refiriéndose a los rarámuri como los corredores por excelencia (Acuña, 2009, p. 341; Bennett y Zingg, 1978 [1935]). Esto ha dado pie, también, para que algunos reflexionemos sobre la concepción rarámuri del cuerpo.

\section{VENIMOS DE ARRIBA Y DE ABAJO}

Los rarámuri que aún no han nacido viven en la casa de Onorúame [el que dicen es Padre], allá en el tercer cielo. Allí viven las almas de los rarámuri, y cuando van a venir a la tierra él las manda y luego nacen para andar aquí sobre el pueblo. Allá viven bien, no sufren, no se enferman ni nada, pues están con nuestro Padre y nuestra Madre. Ellos los cuidan y los crían a todos los rarámuri hasta que se

3 Virgen de Guadalupe y Reyes, respectivamente. 
Un CONCEPTO RARÁMURI DE CUERPO. EXPERIENCIA PRÓXIMA E INTERPRETACIÓN...

los mandan a sus padres acá en la tierra para que ellos los aconsejen (entrevista con José María en Narárachi, Chihuahua, 02/4/2008).

De acuerdo con el relato anterior, los rarámuri que aún no han nacido en la tierra viven en la casa de Onorúame, y míticamente el cuerpo está constituido por la tierra que esta deidad formó y coció para dar origen a los humanos. Asimismo, de acuerdo con lo dicho por los colaboradores, la conformación de un cuerpo se funda en la unión del semen masculino y la sangre femenina. El soplo divino que aporta tres almas al hombre y cuatro a la mujer, complementa la constitución corporal de todo cuanto aparece en el cosmos rarámuri y, al parecer, no solo de los humanos. De allí que los rarámuri vienen de arriba y de abajo.

\section{SA'PÁRA}

Desde la perspectiva del antropólogo, la traducción de "cuerpo" indígena sigue siendo un asunto tan complicado como lo fuera en épocas pasadas para los primeros europeos que intentaron hacer dicha traducción (Surrallés, 2010). ¿Cómo podemos definir hoy el término cuerpo, a partir de los conceptos de experiencia próxima entre los rarámuri? Por ahora diré que la voz rarámuri sa’pára o “carne de" es, quizá, lo más cercano a nuestros términos para llamar al cuerpo; es algo similar al contenido de nuestro término "materia”. Sa’pára se aplica a todo ente corpóreo en el universo de los rarámuri, pero con sus particularidades, como veremos; por ahora, empleamos aquí la palabra materia solo como herramienta heurística. Por cuestiones de espacio no me detengo en toda la terminología empleada por los rarámuri del ARC para nombrar cada parte del cuerpo. Para este aspecto, véase: Acuña (2007, pp. 72-73) y Martínez (2012, p. 22). 
Figura 1. Ubicación de la sierra Tarahumara y la ciudad de Chihuahua, Chih., México.

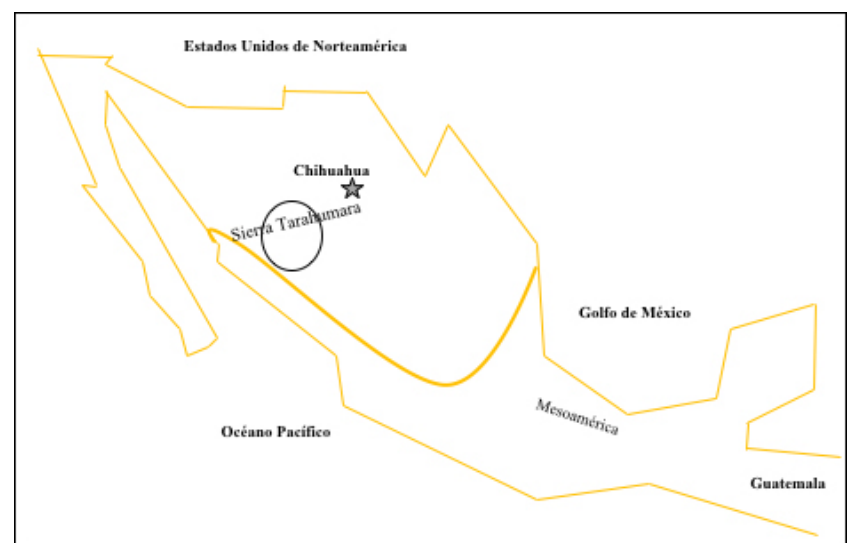

Fuente: elaboración propia (2011).

Surrallés (2010) también ha propuesto tres opciones para el análisis de la noción de cuerpo indígena en América: 1) La mesoamericana, en la cual el cuerpo se interpreta como carne o materia; 2) La centroamericana, en la cual el cuerpo ha sido visto como una dualidad cambiante; y 3) La sudamericana, en la cual el cuerpo se ha definido como volumen, o bien, como la "forma contenida" o "forma continente" (pp. 64-81). Aun cuando la cercanía entre Mesoamérica y el norte de México podría hacernos caer fácilmente en la primera formulación, pienso que todavía estamos por traducir la noción de cuerpo construida desde el norte de México y, particularmente, entre los rarámuri. 
UN CONCEPTO RARÁMURI DE CUERPO. EXPERIENCIA PRÓXIMA E INTERPRETACIÓN...

Figura 2. Ubicación del Alto Río Conchos en el municipio de Carichí, Chih.

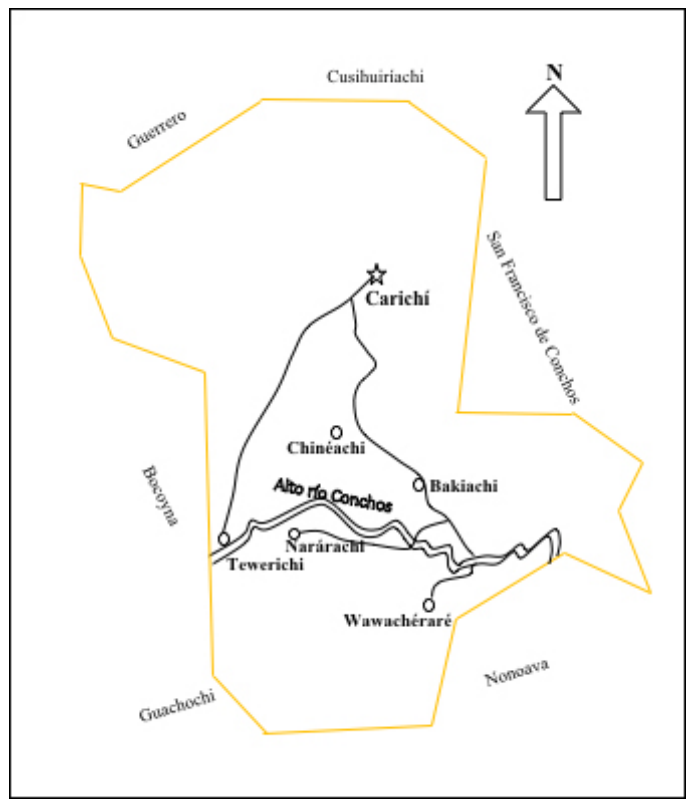

Fuente: elaboración propia (2011).

Por su parte, mis interlocutores emplean el término sa’pára de manera coloquial y sin mayor esfuerzo para expresar la idea de “cuerpo" y, en general, los rarámuri comparten rápidamente un significado. He dicho que con esto se refieren tanto al interior como al exterior, carne y huesos, aunque también parecen incluir el sustrato anímico. ${ }^{4}$ Reitero que estrictamente sa'pára significa "carne de" (Brambila, 1976, p. 506), porque el cuerpo es siempre de alguien y no es materia en abstracto.

Esta "carne de" constituye todo lo que forma parte del cuerpo, porque aun cuando el "alma" (wigá) o la multiplicidad de "almas" (iwigá) no forma parte de este sa'pára, sin las almas el cuerpo de cualquier ser (humano o no humano) no viviría, no existiría. Así como se considera entre los grupos cercanos a los rarámuri (tepe-

4 De sa’pá, "carne" y -ra, sufijo posesivo. El término tiene alguna productividad y existen también las voces sa'pá, sa'paka, sa'péame, pero estas equivalen solo a "carne" humana o animal. 
huanes, pimas y warijíos), la multiplicidad de almas contenidas en los cuerpos humanos es también una idea extendida hasta Sudamérica entre grupos como los chayahuitas del Perú (González, 2010, pp. 244-245), pasando por Mesoamérica (López, 2008 [1980]), y entre los sujetos de mi caso, Merrill (1992) es quien mejor ha estudiado este aspecto.

Algunos interlocutores rarámuri afirman que el cuerpo de uno "es como una casa donde viven las almas, cuando estas se van la casa se cae". Ejemplo de ello ocurre al beber el tesgüino (cerveza de maíz fermentado). Como se ha señalado (Kennedy, 1963, 1970; Rodríguez, 2009), para los rarámuri la bebida colectiva conocida como tesgüinada, resulta ser un evento social de primera importancia. Durante una reunión así, un rarámuri me comentó:

cuando uno toma, ¿a dónde llega primero la bebida? Al corazón, por eso uno se pone alegre, habla más fuerte, canta y hasta baila, y luego el tesgüino sube a la cabeza; luego se revuelve con la sangre y entonces el cuerpo ya no sabe nada. Estás borracho.

Aquí también, como se ha registrado en otra zona cercana, se dice que el olor de la fermentación de la bebida embriagante, que corre por las venas, hace huir a las almas interiores, y de allí la embriaguez (Merrill, 1992).

Sin embargo, para otros rarámuri el alma es como el aire o como sombra (Rodríguez, 2012) y por eso da la impresión de que, para la exégesis indígena, esta forma parte del cuerpo. De este modo, lo que nosotros entendemos por "materia" entre los rarámuri parece tener dos particularidades que la distinguen. Por un lado, es algo concreto, pero por otro tiene también algo de inmaterial. O bien, ¿es el alma rarámuri un cuerpo dentro de otro? Si el alma en el cuerpo es como el ser humano dentro de su casa, entonces el cuerpo rarámuri está conformado por dos materias que forman una sola. Sin embargo, la naturaleza de ambas es diferente, pues una viene de arriba (las almas) y otra se origina abajo (la carne). No obstante, creemos que no se trata de una dualidad cambiante, porque en este caso sa'pára no se refiere al cuerpo simplemente 
Un CONCEPTO RARÁMURI DE CUERPO. EXPERIENCIA PRÓXIMA E INTERPRETACIÓN...

como a la carne y al alma como a un tipo de cuerpo o persona, como sí parece ocurrir en algunas lenguas mayenses como el tzeltal (Surrallés, 2010, p. 74). Carne y alma parecen estar contenidos en la noción sa'pára, pero me atrevo a decir que no se trata de una dicotomía al estilo cartesiano, sino de una dualidad de elementos complementarios que conforman el cuerpo rarámuri y que se distinguen entre sí, como veremos un poco más adelante. Quizá aquí no deberíamos hablar de "cuerpo", sino de cuerpos, en plural. Así como se habla de pluralidad de almas, podríamos hablar de pluralidad de cuerpos. En este sentido,

Nuestro cuerpo es como el de nuestro papá o mamá, porque muchos nos parecemos a ellos y hasta somos igualitos a ellos, y trabajamos igual que ellos. Por eso yo creo que mi cuerpo es parte del cuerpo de mi padre, que ya murió hace tiempo. Es como un pollo que sale del huevo de la gallina, casi siempre salen igualitos que la mamá o el papá (entrevista con José María en Narárachi, Chihuahua, 04/4/2015).

Lo que sugieren estas palabras de José María es que el cuerpo rarámuri no se constituye solo como "un cuerpo", de manera individual, sino que se va formando gracias a la socialización; por ello, podríamos señalar que se trata de "cuerpos". En realidad, no existe "el cuerpo", sino los cuerpos en relación continua y constante. Además del parecido físico a que remite lo dicho explícitamente por José María, entre él y su padre, también sugiere que la relación entre ellos es base de la noción que se tiene del cuerpo. Un trabajo colectivo como rehacer una vivienda o sembrar juntos son formas como los rarámuri se relacionan entre sí, y en este sentido, los cuerpos existen y se van conformando como tales.

\section{DEL CUERPO AL ENTORNO}

El cuerpo rarámuri, esta "materia" tanto femenina como masculina, está conformado por un arriba: la cabeza (mo'ó), de la raíz mo que procede del término moba, es decir, "sobre". La cabeza está en el campo semántico de lo alto y del arriba (repá), y como me señaló 
un joven rarámuri: “por eso le decimos mo'óhinora al cerro más alto de la sierra Tarahumara, pues desde arriba se mira todo, como Ramiro [un rarámuri muy alto] que puede ver todo desde arriba”. Además, este cuerpo está formado por un abajo: los pies (ronó). Los pies corresponden al campo semántico del abajo. Asimismo, los animales son también considerados entre los rarámuri con un cuerpo, como materia. En primer lugar, son considerados como cuerpos al modo en el que para Douglas (1998) en el Levítico: "los animales para ofrecer en sacrificio son cuerpos que representan la rectitud” (p. 209). La rectitud es un ideal perseguido por el rarámuri en tanto que los consejos de sus autoridades son la forma "como los antiguos nos enseñaron”, refiriéndose al buen comportamiento que un rarámuri debe tener en su vida. Parte de este buen comportamiento es dar alimento a Onorúame, ofreciéndole animales domésticos, especialmente vacas y cabras blancas; dicho color representa entre ellos la integridad ideal de un ser humano, cuyo exterior corresponde a un cuerpo bien proporcionado. En segundo lugar, los animales son considerados con mo'ó y ronó, es decir, con "cabeza" y "pies". El arriba y el abajo sobresalen en la consideración de los cuerpos entre los rarámuri del ARC, porque entre los humanos son las partes corporales más significativas en tanto que la cabeza es portadora del natari (pensamiento) y con los pies se produce el movimiento: se corre, camina y danza. De hecho, uno de los significados más generalizados de la voz rarámuri es el de "pies ligeros", dada la importancia que tienen las acciones de caminar, correr y danzar para este pueblo; y como me indican los interlocutores, tanto humanos como no humanos (animales, plantas y algunos astros) poseen pensamiento.

En el cuerpo humano la frontera entre estos dos espacios es el sikú, es decir, el "ombligo". Sikú, también traducido como "rincón”, que remite, a su vez, a los centros y puntos que articulan, en el espacio territorial, el mundo de arriba con el de abajo, es decir, las cuevas (ombligos del mundo); y así se manifiesta la proyección del cuerpo humano en el entorno. Pero esta proyección queda aún más clara en la consideración rarámuri sobre las cosas más inmediatas, y así, por ejemplo, a los espejos laterales de los automóviles 
Un CONCEPTO RARÁMURI DE CUERPO. EXPERIENCIA PRÓXIMA E INTERPRETACIÓN...

los nombran nakároa, "orejas"; a la cuchara la llaman seká, "mano"; a las bolsas de plástico, chiwawira, "escroto”; sin olvidar lo dicho en la introducción acerca de múltiples voces que parecen proyectar algunos rasgos del cuerpo humano en el entorno.

\section{DEL CUERPO AL COSMOS}

En su Compendio de la lengua de los tarahumares y guazapares, Guadalajara (1683) registró cuatro modos distintos de contar empleados por los rarámuri del siglo xvir: "Ovatro modos ay de contar, vno es halta feis otro hafta diez, otro hafta doze, otro halta veinte" (Rodríguez, 2010, p. 233). Todos se contaban con los dedos de las manos y los pies. El veinte se traduce como bieré rejói, es decir, "un hombre", stricto sensu, "un cuerpo"; y aunque los rarámuri del siglo xxI de nuestra región de estudio ya no emplean este término, pero sí lo comprenden, este sigue siendo usual en la región de la barranca. Hoy en día, los rarámuri del ARC utilizan los términos makoi y osá makoi, es decir, "diez" y "dos veces diez" (o veinte), sugiriendo dichas voces que la suma de los dedos de las manos y la suma tanto de estos como de los dedos de los pies conservan la idea antigua de considerar las extremidades del cuerpo humano. Esto sugiere que la observación de lo múltiple, es decir, del universo, debió partir de la observación de lo simple; y así, para contar las cosas se partió de la primera referencia que ofrece la experiencia humana de la unidad inmediata: el cuerpo. Un modo probable de iniciar proyectando el cuerpo al cosmos, "porque es imposible concebir la pluralidad sin la unidad. Si lo uno no existe, las otras cosas [...] no son concebidas" (Parménides, 1871, p. 272).

Por otro lado, como hemos visto, existe terminología que nos indica que algunas partes del cuerpo son afines a otros elementos de la naturaleza y así el cuerpo humano comparte con "otros" algunos caracteres. No es extraño, pues, que el cuerpo que aparece ante los sentidos sea una masa física (carne) que ocupa, incluso, una posición en el universo, como lo sugieren los cinco verbos de posición que se emplean para referirse al modo de estar de los cuerpos en el mundo (sentado, parado, tendido, contenido y 
pegado o en cuatripié). Se trata de una proyección del cuerpo humano sobre la naturaleza, ordenamiento con base en una analogía cuerpo-cosmos. En este sentido, no es extraño que los rarámuri de regiones vecinas al ARC consideren la tierra como un cuerpo:

sus piernas y sus brazos, su corazón, su carne, sus huesos y su sangre [son] los inmensos árboles del bosque, y los pastos de la pradera son su cabello, la tierra es su carne, las piedras de los arroyos y las rocas de las montañas son sus huesos, el aire es su aliento (Moreno, s.a., p. 5).

Por medio de la analogía, de acuerdo con Foucault (2014 [1966]), pueden relacionarse todas las figuras del mundo. Y esto es posible gracias a que existe un punto a través del cual pasan todas las analogías, y estas pueden invertirse sin alterarse. Este punto es el hombre, dice Foucault (2014 [1966]) parafraseando a Crollius, pues el ser humano es quien está:

En proporción con el cielo, y también con los animales y las plantas, lo mismo que con la tierra, los metales, las estalactitas o las tormentas. Erguido entre las faces del mundo, tiene relación con el firmamento [...] pero equilibra todas estas relaciones y se las reencuentra, similares, en la analogía del animal humano con la tierra que habita: su carne es gleba; sus huesos, rocas; sus venas, grandes ríos; su vejiga, el mar y sus siete miembros principales, los siete metales que se ocultan en el fondo de las minas (p. 40).

Y más precisamente en la antropología moderna es Mary Douglas (1973) quien ofrece esta interpretación sobre el cuerpo, al considerar que "el cuerpo es un modelo que puede servir para representar cualquier frontera precaria o amenazada” (p. 156), significando, con ello, que el cuerpo es la referencia más común para determinar otras estructuras complejas como el cosmos mismo. De la misma manera, pero desde un punto de vista estructuralista, Galinier (1990) consideró que uno de los axiomas que constituyen el modelo de representación del mundo otomí, otro grupo indígena 
Un CONCEPTO RARÁMURI DE CUERPO. EXPERIENCIA PRÓXIMA E INTERPRETACIÓN...

mexicano, es este: "el universo es una réplica a gran escala del cuerpo humano” (p. 682). Como veremos, esto último nos indica ya una diferenciación entre la visión norteña y la mesoamericana sobre el cuerpo, porque entre los rarámuri falta considerar la noción de cuerpo como resultado de representaciones sociales y procesos históricos.

\section{DE LA SOCIEDAD AL CUERPO}

Hemos señalado ya que para los rarámuri el cuerpo se constituye también socialmente. El objetivo, parece, es hacer que una persona exista y desarrolle su vida con los otros. Es lo que sugiere el hecho de que las mujeres deseen con toda su fuerza, durante el embarazo, que sus hijos resulten con cuerpos completos, ya que, de no ser así, un neonato con cuerpo incompleto o, incluso, falto de sus facultades mentales (incompleto de almas), no sea deseado. En mi primera estancia en el ARC (1999-2003) escuché el testimonio de una religiosa, quien en la década de 1970 recogió a un infante cuyo defecto físico (manos incompletas y sin dedos) hizo que sus padres lo abandonaran en un cerro para que se muriera. Se trata de una práctica que, como me lo han dicho otros agentes de pastoral de la Diócesis de la Tarahumara, quienes por años han vivido en comunidades indígenas de la región, quizá hoy es posible que se realice en algunas zonas apartadas, aunque reconocen que no han sabido de algún caso así por décadas. Esto no deja de remitir al reporte hecho por Bennett y Zingg (1978 [1935]) en los años treinta del siglo pasado, que narra sobre el producto de un incesto: "la joven mató al bebé dejándolo caer sobre rocas" (p. 360). La razón más fuerte que, parece, tuvieron los rarámuri para llevar a cabo estas acciones en el pasado, es que si un infante nacía carente de alguna parte de su cuerpo (o sus facultades mentales) sin duda sufriría. En principio, ninguna mujer aceptaría casarse con un hombre que no fuera capaz de sembrar, usar el hacha en el corte de la leña, es decir, que no pudiera emplear sus manos, piernas u otra parte del cuerpo para trabajar; así como durante su infancia y juventud el sujeto no sería capaz de valerse por sí 
mismo y representaría una carga para "todos". Y lo mismo, o quizá peor, sería para una mujer carente, por ejemplo, de alguna de sus extremidades. ¿Y qué decir de la necesidad de un cuerpo completo en un grupo en el que danzar (sobre todo entre los hombres) obedece a una obligación tanto moral como a un deseo personal de socializar? Sin cuerpo no hay danzante, porque aquel es la herramienta técnica de quien danza; y lo mismo ocurre con aquellas corredoras de aro (mujeres) y corredores de bola (hombres), quienes precisan de un cuerpo completo, proporcionado y potente, para resistir los periplos convertidos en enormes distancias que por horas deben recorrer en estos eventos deportivos propiamente indígenas. En este sentido, encontramos que el cuerpo es tanto una expresión de lo social como del poder político, entendido este como la exigencia social; el cuerpo es también "aquello que el efecto del poder constituye como sujeto” (Foucault, 1976, p. 23).

El cuerpo rarámuri es, además, el resultado de la imperiosa necesidad de trabajar para sobrevivir en un medio agreste como la sierra, que, aun cuando los rarámuri han aprendido históricamente a vivir en ella, tiene épocas (sequías y malas cosechas, nevadas, etcétera) en las cuales deben migrar para buscar otras formas de sobrevivencia (cosecha de manzana y tomate, construcción de viviendas y cuidado de ranchos, entre mestizos, principalmente). Un autor que describió el cuerpo de los rarámuri, y que se admiró de la destreza en su uso fue Lumholtz (1981 [1902]). Este explorador señaló cómo los rarámuri son excelentes en el empleo de, por ejemplo, sus manos para el trabajo: "tiene la tribu innegablemente cierto don para la mecánica, pues todos disponen de gran destreza de dedos que les permite hacer bien las cosas" (t. I, p. 242). Hoy en día yo no diría algo distinto, ya que los rarámuri trabajan la artesanía y fabrican herramientas y utensilios cuyos detalles siempre requieren una verdadera destreza manual.

Como ocurre en otras sociedades, entre los rarámuri un cuerpo incompleto no podría subsistir plenamente. El caso de un joven que conocí en el municipio de Carichí, Chihuahua, quien nació con un muñón al final de su brazo derecho, extremidad que esconde permanentemente bajo su ropa y quien siempre se aísla en 
Un CONCEPTO RARÁMURI DE CUERPO. EXPERIENCIA PRÓXIMA E INTERPRETACIÓN...

Figura 3. Danzantes fariseos de Narárachi, Semana Santa 2011.

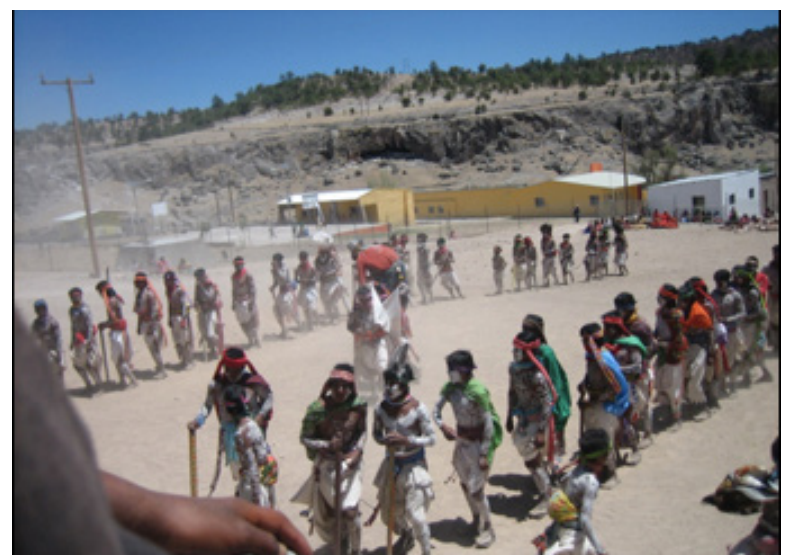

Fuente: archivo propio (2011).

los momentos de trabajar colectivamente, demuestra dos cosas importantes ante lo dicho párrafos arriba: 1) Que el cuerpo sigue siendo una expresión de la exigencia social y de la imperiosa necesidad del trabajo para sobrevivir; y, 2) Que el deshacerse de un infante nacido con un cuerpo incompleto es una práctica desaparecida, o bien, sugiere que, quizá, se practicó alguna vez debido a casos extremos. Este caso permite ver, también, que la exigencia social impone un modo de presentar el propio cuerpo rarámuri. Un cuerpo completo, proporcionado en sus distintas partes, sa'péame (carnoso), como señalan los colaboradores, es decir, grueso (gordito), es motivo de orgullo en hombres y mujeres. Se trata de cuerpos cuya presentación con una actitud gallarda, alegre y que tiende a la risa continuamente, manifiesta una excelente disposición a la vida. Con todo, el cuerpo rarámuri es también producto de una historia de encuentros a lo largo de la historia del grupo.

\section{EL CUERPO CAMBIA CON LA HISTORIA}

Para los rarámuri el cuerpo no siempre es el mismo, pues eventualmente muta, y la relación histórica con el catolicismo hizo de la ritualidad un espacio donde los cuerpos se transforman. Y así, 
por ejemplo, los danzantes integran a su cuerpo la pintura que los caracterizará por los días del noríroachi o "cuando se dan vueltas" (Semana Santa del calendario tridentino, tiempo en el cual se danza representando la lucha del bien y el mal entre dos bandos contrarios: los fariseos, enemigos de Cristo, y los soldados, amigos de Cristo). La pintura no es un simple adorno, sino que es considerada por ellos como parte del cuerpo, el cual, así, tiene un elemento más, pero ¿qué le añade al cuerpo rarámuri? Quien porta la pintura es considerado por los demás como aquel que cumple con la norma establecida (y anhelada) de danzar, para que el mundo no se caiga; "si dejamos de danzar el mundo se caería”, dicen las autoridades locales en sus discursos públicos.

La pintura representa, para quienes la portan, parte de la identidad del cuerpo que se requiere emplear en esta época del año, "sin la pintura no se puede ser fariseo", dice un danzante. La transformación del cuerpo se hace a través de la pintura y otros adornos como, eventualmente y quizá cada vez menos, el sombrero con plumas de guajolote y la espada de madera convertida en la prolongación del brazo de los danzantes, quienes emplean sus brazos para luchar simbólicamente contra el grupo adversario. Todos estos elementos (pintura, sombrero con plumas y espada), se dice, sirven para fortalecer al cuerpo, y así quedar completo en un importante tiempo ritual. De este modo, el ritual mismo queda impreso en el cuerpo portador de una transformación, no solo biológica sino también social, basada en la historia de encuentros que el grupo ha tenido con otros modos de pensar originados en otras latitudes.

\section{CUERPO Y PERSONA}

Los rarámuri identifican el cuerpo, cuya noción he venido esbozando hasta ahora, pero identifican también personas. Entre ellos, el cuerpo designa a "otro", pero el trato con el otro parece ser el espacio de identificación de los sujetos, ya que ellos son quienes tienen la capacidad de pensar (el nátari). No obstante, la concepción de persona parece tener en su base la concepción de cuerpo, 
Un CONCEPTO RARÁMURI DE CUERPO. EXPERIENCIA PRÓXIMA E INTERPRETACIÓN...

porque, como concepto de experiencia próxima, la persona apenas se distingue del cuerpo. Es quizá, por ello, que los rarámuri sugieren que la persona es, ante todo, un cuerpo animado y que un cuerpo por sí solo no es persona. De este modo, el cuerpo y la multiplicidad de almas que hacen aparecer la idea del "pensamiento" conforman a la persona, ya que las almas, por otro lado, son el origen del movimiento de un cuerpo. Esto nos ayuda a entender mejor el concepto de materia rarámuri, porque, entonces, descubrimos que, desde este punto de vista, no podemos hablar sino de cuerpo/persona y no de cuerpo y persona, aunque la distinción aquí es empleada de un modo heurístico. De esta manera, el concepto de cuerpo nos ha dejado en el umbral del concepto de persona.

\section{CONCLUSIONES}

Hasta aquí hemos intentado hacer una descripción densa en términos de Geertz (2005 [1973]), para quien la etnografía no es densa "en el sentido de hacer una descripción de las costumbres o las conductas humanas incomprensible sino de explicar los códigos de entendimiento entre las personas de un mismo grupo" (pp. 21 y 24). De este modo, hemos valorado la relevancia de una serie de conceptos de experiencia próxima en relación con diversos aspectos culturales que tienen que ver con el cuerpo y sus usos (sistema lengua-cultura); es decir, hemos tratado de captar las características de la cultura no solo a través de la observación, sino también del simbolismo lingüístico que, de acuerdo con Sapir (1956: 68), "hace inteligibles esas características a la sociedad".

Con estos códigos de entendimiento que se enmarcan en el lenguaje común y ciertos usos especializados de las palabras usuales que constituyen el principal vehículo de las representaciones comunes de la sociedad rarámuri, encontramos que el cuerpo no es solo una entidad biológica, no es solo materia. Hemos visto que el concepto occidental de "materia" estorba para concluir qué es, finalmente, el cuerpo entre los rarámuri del ARC, aun cuando teóricamente hablamos de una trama amplia de significados. 
Entre estos rarámuri existe una tendencia a tomar como modelo la corporeidad inmediata y proyectarla al cosmos. Se aplica, así, una analogía, es decir, se toma como modelo morfológico el cuerpo. Así lo muestran los diversos elementos del entorno natural o el hecho de que diferentes elementos del cosmos sean considerados con características humanas. Sin embargo, ocurre también lo contrario; es decir, no es extraña la consideración de que el cuerpo sea el resultado de representaciones de distinto tipo, como el hecho de que sea expresión de las relaciones y exigencias sociales, políticas y rituales, y que cargue con responsabilidades y enseñanzas históricas; así como que en él se proyecte la vida que se desarrolla en un medioambiente agreste como la sierra Tarahumara, donde se requiere un cuerpo completo, fuerte y capacitado para el trabajo ante la exigencia para sobrevivir.

Por lo tanto, desde algunos conceptos de experiencia próxima, el cuerpo es, por un lado, fuente de representaciones del cosmos. De este modo, podemos también considerar al cuerpo como una estructura en la cual "las funciones de sus partes diferentes y sus relaciones ofrecen una fuente de símbolos a otras estructuras complejas" (Douglas, 1973, p. 156). Por otro lado, el cuerpo también es concebido aquí como producto de representaciones, sobre todo como producto de las relaciones sociales e históricas en las cuales sobresale el poder político, entendido este como el ejercicio de coerción explícita e implícita desde la sociedad y las instituciones sociales establecidas, como puede ser la ritualidad impuesta a través de la historia o el matrimonio que exige una pareja completa en todo sentido, incluyendo el cuerpo. Como se ha sugerido, el poder político tiene "el papel de inscribir perpetuamente, a través de una especie de guerra silenciosa, la relación de fuerzas en las instituciones [...] hasta en los cuerpos de unos y otros" (Foucault, 1976, p. 24).

Un aspecto relevante más, es que el cuerpo concebido por los rarámuri del $\mathrm{ARC}$, se diferencia de la noción de cuerpo mesoamericana. Entre los norteños, por una parte, el cosmos es solo parcialmente considerado como una réplica del cuerpo y no como sucede entre algunos grupos actuales de Mesoamérica, para quie- 
Un CONCEPTO RARÁMURI DE CUERPO. EXPERIENCIA PRÓXIMA E INTERPRETACIÓN...

nes el cosmos es una réplica completa del cuerpo humano (para los otomíes, por ejemplo). Hemos visto que tampoco se trata de una dualidad cambiante, como sucede en el pensamiento mayense, porque el concepto sa’pára rarámuri expresa más que solo la fisicalidad, aunque distingue entre lo físico y el sustrato anímico.

Ante el estorbo, más que ayuda del concepto "materia", queda la tarea de encontrar el significado propio de este cuerpo (y la persona) base de la sociedad rarámuri. Por ahora sugiero que el término sa’pára puede ayudarnos a entender esta noción, pero apenas nos hemos acercado a comprenderla.

Finalmente, encuentro que las categorías paradigmáticas "arriba” y "abajo" aparecen en la noción de cuerpo rarámuri, que por cuestiones metodológicas seguimos llamando así. De acuerdo con el relato inicial de José María, la multiplicidad de almas formadas por una materia inaprehensible, se origina y termina en el arriba; en contraposición con la materia del cuerpo, materia concreta y aprehensible, que nace de la tierra y permanece en esta hasta el final de su vida; es decir, en el abajo. De este modo, la noción de "cuerpo" parece corresponder al abajo y la de "persona" parecería corresponder al arriba. La importancia de este eje (arriba-abajo) radica en que, hacia dentro de la comunidad rarámuri, se refleja una notable consideración de los sexos masculino y femenino en este orden jerárquico, que muy probablemente se proyecta, incluso, en una jerarquización social en donde los hombres (de mayores a menores) tienen una mayor relevancia que las mujeres en el acontecer social público del grupo. Sin embargo, habría que explorar si esto es igual en el ámbito de lo privado y si aquí se cumple el principio de división fundamental entre lo masculino activo y lo femenino pasivo, el cual "crea, organiza, expresa y dirige el deseo masculino de posesión y dominación”, señalado por Bourdieu (2000: 19).

\section{BIBLIOGRAFÍA}

Acuña, A. (2009). Usos del cuerpo en la construcción de la persona rarámuri (Chihuahua, México). Gazeta de Antropología, 25(2), 
artículo 36. Recuperado el 4 de febrero de 2016, de http://hdl. handle.net/10481/6913.

- (2007). La construcción cultural del cuerpo en la sociedad rarámuri de la sierra Tarahumara. Quito: Abya Yala.

Bennett, W., \& Zingg, R. (1978 [1935]). Los tarahumaras: una tribu india del norte de México (Clásicos de la Antropología 6). México: Instituto Nacional Indigenista.

Bourdieu P. (2000). Una imagen aumentada (la construcción social de los cuerpos). En Pierre Bourdieu La dominación masculina. Traducción de Joaquín Jordá. España: Editorial Anagrama Barcelona, pp. 8-41. Recuperado el 10 de octubre de 2018 http://www.nomasviolenciacontramujeres.cl/wp-content/ uploads/2015/09/Bondiu-Pierre-la-dominacion-masculina.pdf.

Bourdieu, P. (2008). La ruptura: el hecho se conquista contra la ilusión del saber inmediato. En P. Bourdieu, J. C. Chamboredon, \& J. C. Passeron (Eds.), El oficio del sociólogo: presupuestos epistemológicos. México: Siglo XXI.

Brambila, D. (1976). Diccionario rarámuri-castellano. México: Obra Nacional de la Buena Prensa.

Douglas, M. (1998). Estilos de pensar. España: Gedisa.

---- (1973). Pureza y peligro: un análisis de los conceptos de contaminación y tabú. Madrid: Siglo XXI de España Editores, S. A. Durkheim, E., \& Mauss, M. (1971 [1903]). De ciertas formas primitivas de clasificación. Contribución al estudio de las representaciones colectivas. Obras II: Institución y culto. Barcelona: Seix Barral.

Foucault, M. (2014 [1966]). Las palabras y las cosas: una arqueología de las ciencias humanas. México: Siglo XXI.

---- (1976). Genealogía del racismo. Argentina: Altamira.

Galinier, J. (1990). La mitad del mundo: cuerpo y cosmos en los rituales otomíes (colección Antropología y Etnología). México: Centro de Estudios Mexicanos y Centroamericanos/Instituto Nacional Indigenista/Universidad Nacional Autónoma de México.

Geertz, C. (2005 [1973]). La interpretación de las culturas. España: Gedisa. 
Un CONCEPTO RARÁMURI DE CUERPO. EXPERIENCIA PRÓXIMA E INTERPRETACIÓN...

----- (2004). Conocimiento local: ensayos sobre la interpretación de las culturas. Barcelona: Paidós Básica.

González, L. (1982). Tarahumara, la sierra y el hombre. México: Secretaría de Educación Pública/Fondo de Cultura Económica.

---- (1987). Crónicas de la sierra Tarahumara. México: Secretaría de Educación Pública.

González, M. (2010). El cuerpo como referente del universo chayahuita: aproximación a una teoría de la persona. En M. Gutiérrez Estévez, \& P. Pitarch (Eds.), Retóricas del cuerpo amerindio. Madrid: Iberoamericana-Vervuert.

Guadalajara, T. (1683). Gramática de la lengua de los tarahumares y guazapares. En D. Fernández de León (Ed). México: Puebla de los Ángeles.

Guillén, H., \& Martínez, I. (2004). Del cuerpo a la persona: ensayo sobre una noción rarámuri. México: Universidad Autónoma del Estado de Morelos (mimeo).

Instituto Nacional de Estadística y Geografía (Inegi). Encuesta Intercensal 2015. Recuperado el 14 de febrero de 2017, de http://www3.inegi.org.mx/sistemas/TabuladosBasicos/Default. aspx?c=27303\&s=est

Kennedy, J. (1970). Inápuchi: una comunidad tarahumara gentil. México: Instituto Indigenista Interamericano.

---- (1963). Texgüino Complex: The Role of Beer in Tarahumara Culture. American Anthropologist, 65, 620-640.

Le Bretón, D. (2002). Antropología del cuerpo y modernidad (colección Cultura y Sociedad). Buenos Aires: Nueva Visión.

López, A. (2008 [1980]). Cuerpo humano e ideología: las concepciones de los antiguos nahuas. (serie Antropológica 39, 2 vols.). México: Universidad Nacional Autónoma de México- Instituto de Investigaciones Antropológicas.

Lumholtz, C. (1981 [1902]). El México desconocido (T. I, colección Clásicos de la Antropología 11). México: Instituto Nacional Indigenista.

Martínez, I. (2012). Alteridad, multiplicidad y reversibilidad en clave rarámuri: crónica de un viaje por la antropología del otro. Uni- 
versidad Nacional Autónoma de México-Instituto de Investigaciones Antropológicas (mimeo).

----- (2008). Los caminos rarámuri: persona y cosmos en el noroeste de México. Universidad Nacional Autónoma de México-Instituto de Investigaciones Antropológicas (mimeo).

Merrill, W. (1992). Almas rarámuris. México: Consejo Nacional para la Cultura y las Artes/Instituto Nacional Indigenista.

Moreno, J. (s.a.). La Madre Tierra. En J. R. Moreno Gutiérrez (Comp.), Benebóo ki'yá e'peréame ra'ícháara: aprendamos relatos de los antepasados. Chihuahua: Cedain, A. C.

Parménides (1871). Platón, obras completas, 4. En P. de Azcárate (Ed.). Madrid: Edición particular.

Rodríguez, A. (2015). Conceptos próximos e interpretación distante: espacio y tiempo en el pensamiento rarámuri. Anales de Antropología, 49(2), 73-100.

(2012). Los rimuká, "hilos de vida y muerte", elemento cardinal en la etiología rarámuri. En A. Gutiérrez del Ángel (Ed.), Hilando al norte: nudos, redes, vestidos, textiles (pp. 235-256). México: El Colegio de San Luis/El Colegio de la Frontera Norte. ---- (2010). Gramática tarahumara. Ciudad Juárez: Universidad Autónoma de Ciudad Juárez/Universidad Autónoma de Chihuahua/Consejo Nacional para la Cultura y las Artes/Instituto Chihuahuense de la Cultura.

----- (2009). Reunión y bebida colectiva entre los rarámuri (la tesgüinada): una institución difícil de comprender. En V. Orozco (Coord.), Chihuahua Hoy 2009. México: Universidad Autónoma de Ciudad Juárez/Universidad Autónoma de Chihuahua/Instituto Chihuahuense de la Cultura.

Sapir, E. (1956). Culture, Language, and Personality: Selected ESsays. En D. Goodman Mandelbaum (Ed.). Berkeley: University of California Press.

Surrallés, A. (2010). La retórica de traducir "cuerpo". En M. Gutiérrez Estévez, \& P. Pitarch (Eds.), Retóricas del cuerpo amerindio. Madrid: Iberoamericana-Vervuert. 